Engineering.

This reduces the number somewhat and includes one subject not included in your classification. I believe that the time has come when we must have technical journals in subjects pertaining to agriculture just as they have in all other branches of scientific work.

Professor J. W. Gilmore writes:

This scheme seems to me eminently feasible and I believe is a distinct step in arranging and systematizing our station literature. I would like to hear a discussion, however, along three lines at least:

1. Scheme of classification.

2. Whether station workers might not receive any or all of the series free on request.

3. What may be the attitude of the now-established scientific journals toward the scheme.

Would it be well to invite discussion along these lines?

The methods and means of publication for scientific station matter is a subject in which all experiment-station investigators are vitally interested, and the writer has thought it desirable to publish his thoughts on the subject, hoping to stimulate a general discussion out of which sentiment may crystallize so that some advanced step may soon be taken by the station authorities.

\section{CORNELI UNIVERSITY}

\section{H. J. WEBBER}

\section{ON THE EFFECTS OF MAGNESIUM SULPHATE ON PLANTS}

In the issue of Science of August 16, Professor William J. Gies publishes a letter, in which my refutation of an unjust attack is subjected to an analysis which I cannot regard as going to the essential point. I must, therefore, once more and more distinctly state that my inferences as to the poisonous action of magnesium sulphate on plants can, of course, only relate to the conditions of my experiments and that $I$ nowhere have made the assertion that these poisonous actions would be observed also at still higher dilutions than those I had used, for I was very well acquainted with the truth that the action of a poisonous substance decreases with the dilution and that beyond a certain dilution even a stimulating action can take place.
I have further pointed out that the poisonous effects of that salt are modified by the lime content of the cells; the more lime there is present in the cells, the more magnesium sulphate will be required to exert a poisonous action. From this standpoint my own observation on the stimulating action of magnesium sulphate ${ }^{1}$ under certain conditions, becomes intelligible.

Oscar Loew

\section{SPECIAL ARTICLES}

\section{THE SPARK CHRONOSCOPE}

EIGHT years ago I published a description of a new chronoscope in a technical monograph. Eight years of continued use, in which the instrument has been tested for convenience, durability, adaptation and accuracy, give such assurance of satisfaction that $I$ am moved to bring the instrument to the attention of a wider circle of scientists through the columns of this journal. I would especially invite comparison with other instruments on the three fundamental qualities of accuracy, economy in operation and adaptability.

The following brief description is essentially an extract from the original account in The University of Iowa Studies in Psychology, Vol. II., p. $155 \mathrm{ff}$.

Of the hitherto known forms of apparatus for measuring short intervals of time, the graphic spark apparatus is the most accurate and the pendulum apparatus the most convenient. In the chronoscope that is shown in the accompanying figure, the spark method of recording is combined with the pendulum action.

The cut is reduced to a scale one sixth of the size of the apparatus. The pendulum is shown in the starting position. The lower bob terminates in a knife edge which rests upon the projecting edge of a mechanical release key. The action of this key is soundless and gives the pendulum no impetus in either direction. On the other side of the apparatus is a spring key which catches the pendulum at the

${ }^{1}$ Cf. "Flora," 1893; observations on the growth of the roots of Tradescantia, in my article on the "Physiological Functions of Lime and Magnesia." 
end of the swing. When the pendulum is released from this, it swings back with little assistance to the starting point and makes all necessary adjustments automatically. On the

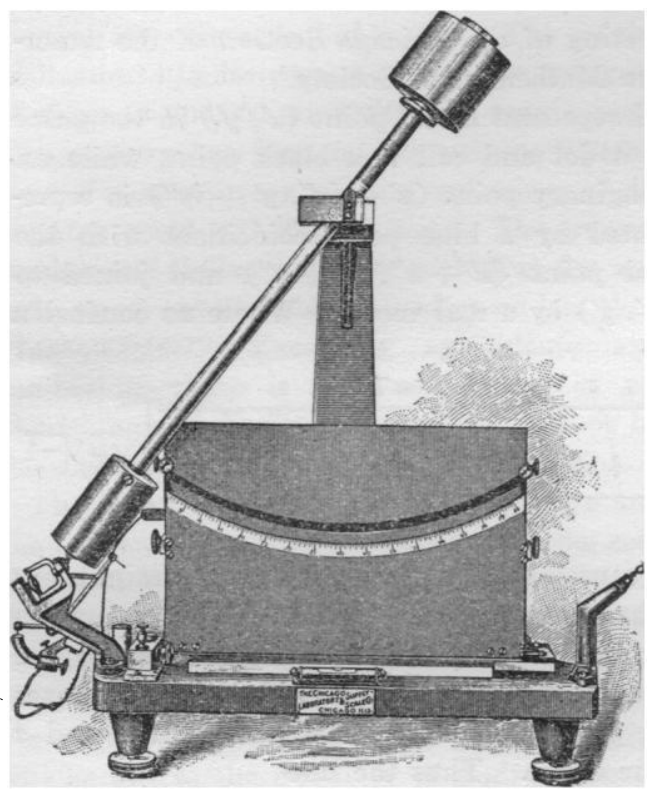

back of the lower bob is an index point which runs at the upper edge of the scale and serves as a spark point.

The record is made upon a smoked paper which is seen through the slit above the scale. This paper is stretched upon two rollers; it also rests upon an insulated metal plate which serves as an electrode and keeps the paper straight and smooth back of the scale. Back of this plate is a third roller by means of which the tension of the paper may be adjusted. The paper support is built on a carriage so that it may be removed and replaced without disturbing the rest of the apparatus. In preparing the paper this carriage is removed and the paper is smoked as on an ordinary kymograph drum. As a complete record consists in a single spark which may be recorded at once, several hundred records may be made with one preparation of the paper. The paper is moved, as needed, by a thumb screw at one end of the upper roller.

In reaction experiments the stimulus is given automatically by the apparatus when the pendulum indicator passes the zero point on the scale. A double rocking lever at this point makes one circuit and breaks another, either of which may be used in giving the stimulus. These contacts are adjustable platinum and mercury contacts and their adjustment may be verified by direct sight. The closing or opening of the circuit is soundless, and the stopping of the lever in a soft rubber clutch makes no sound that can be heard a few feet away.

The reaction, or termination of the interval to be measured, is indicated by a spark on the sensitive paper at the edge of the scale. The spark is produced by interrupting the primary circuit of an ordinary induction coil. One secondary terminal is connected with the insulated plate on which the paper rests and the other is connected with the body of the apparatus. The point of the pendulum indicator is the nearest metal to the plate; therefore the spark flies from this point, through the sensitive paper, to the plate.

The scale is graduated empirically by the most reliable graphic method into hundredths of a second, and each unit is divided into halves. The average space of one unit is 5 $\mathrm{mm}$. on the arc of the scale. With this adjustment the scale covers 0.80 sec. and the records are read in half-hundredths with ease and accuracy. This division is the most convenient and appropriate to use in reaction experiments. The variation in the movement of the pendulum is negligible because the pendulum is carefully constructed and balanced and moves without friction. The variation in the make contact is also negligible because the platinum terminal moves much faster than the pendulum indicator. The spark tends to take the shortest course between the point and the plate, but it may be deflected. The maximum distance between the spark point and the paper is $1 \mathrm{~mm}$. The maximum deflection of the spark may be estimated to be about $45^{\circ}$. That amount of deflection is not liable to occur for the maximum distance, but if it did the maximum variation would be $\pm 1 \mathrm{~mm}$. on the scale, which is equal, on the 
average, to \pm 0.002 sec. The average distance between the spark point and the paper is about .5 mm. and the average angle of deflection of the spark is less than half of $45^{\circ}$; therefore the average variation in the spark is less than \pm 0.001 sec.

The chronoscope may be adapted for the measurement of longer intervals, as in the study of association, by two minor changes which can be made in a minute. A small weight is fastened on the top of the upper bob. This makes the pendulum swing so slowly that it takes three seconds to cover the arc of the scale. A corresponding scale, graduated empirically in hundredths of a second, is clamped over the regular scale. The accuracy is nearly proportional to the speed of the pendulum.

Similarly, if there should be a demand for finer readings than those obtained by the standard adjustment, an extra weight may be placed on the lower bob that will cause the pendulum to cover the arc of the scale, for example, in one third of a second. If the corresponding scale is graduated in thousandths of a second each unit will occupy, on the average, $1 \mathrm{~mm}$. of space. The degree of accuracy will be nearly proportional to the speed, because the latent time of the spark is negligible and the action is frictionless.

Much of the value of a chronoscope lies in its adaptation to the attachment of a variety of accessories. The possession of the soundless make and break contacts for the stimulus circuit makes it possible to connect all sorts of electric stimulus apparatus, such as the telephone receiver, the touch key, the tachistoscopes, etc.

For regulating time-exposures, a movable pendulum contact is attached to the front of the base and adjusted, by reference to the scale, for any desired length of exposure from a hundredth of a second to three seconds. This contact may be used either as a make or break and the circuit may be completed either through the make or the break of the stimulus contacts.

The UNIVERsITy OF JowA

$$
\text { C. E. Seashore }
$$

\section{A VECTOR DIAGRAM}

Apropos of Carl Barus's interesting note in ScIEnce of August 2, p. 149, it may not be amiss to call attention to a representation that I used in a communication to the March meeting of the Chicago Section of the American Mathematical Society. ${ }^{1}$

I represent a real point $\left(x^{\prime}, y^{\prime}\right)$ in the plane by a dot and call it a black point, while an imaginary point $\left(x^{\prime}+i x^{\prime \prime}, y^{\prime}+i y^{\prime \prime}\right)$ is represented by a blue point coincident with the real point $\left(x^{\prime}+x^{\prime \prime}, y^{\prime}+y^{\prime \prime}\right)$ and joined to $\left(x^{\prime}, y^{\prime}\right)$ by a real vector. Where no confusion

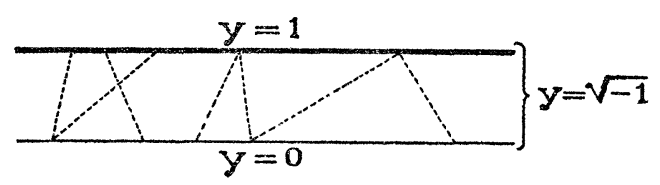

is caused the real vector is drawn straight, but otherwise it may be curved, it being understood that the direction is determined by the end points. Furthermore, if the vector moves its end points describe a black curve and a blue curve. Thus the line

$$
y=\sqrt{-1}
$$

is represented by joining every point in the black line

$$
y=0
$$

to every point in the blue line

$$
y=1 \text {. }
$$

In the accompanying diagram the "blue line" is drawn heavy, the "red lines" broken.

Ellery W. Davis

\section{QUOTATIONS}

LIVING ON OUR CAPITAL

THe passion to beat our records in material advancement tends to blind the thought to the fact that we are rapidly consuming the very fundamental resources on which the prosperity of the country rests. Without doubt the timber supply of the United States is disappearing far more rapidly than any increment of growth. The treatment of the soil in much

${ }^{1}$ Bulletin of the American Mathematical Society, June, 1907, p. 436. 